\title{
Favorable prognostic role of tropomodulins in neuroblastoma
}

\author{
Paola Bettinsoli ${ }^{1}$, Giulia Ferrari-Toninelli ${ }^{1}$, Sara Anna Bonini ${ }^{1}$, Michela Guarienti ${ }^{1}$, \\ Davide Cangelosi' ${ }^{2}$, Luigi Varesioº ${ }^{2}$ and Maurizio Memo $^{1}$ \\ ${ }^{1}$ Department of Molecular and Translational Medicine, University of Brescia Medical School, Brescia, Italy \\ ${ }^{2}$ Laboratory of Molecular Biology, Giannina Gaslini Institute, Genova, Italy \\ Correspondence to: Paola Bettinsoli, email: bettypaola@gmail.com \\ Keywords: neuroblastoma; tropomodulins; favorable prognostic biomarkers; overall survival probability; therapeutic strategies \\ Received: September 15, $2017 \quad$ Accepted: May 07, $2018 \quad$ Published: June 05, 2018 \\ Copyright: Bettinsoli et al. This is an open-access article distributed under the terms of the Creative Commons Attribution License \\ 3.0 (CC BY 3.0), which permits unrestricted use, distribution, and reproduction in any medium, provided the original author and \\ source are credited.
}

\section{ABSTRACT}

Neuroblastoma is a pediatric tumor of the sympatoadrenal lineage of the neural crest characterized by high molecular and clinical heterogeneity, which are the main causes of the poor response to standard multimodal therapy. The identification of new and selective biomarkers is important to improve our knowledge on the mechanisms of neuroblastoma progression and to find the targets for innovative cancer therapies. This study identifies a positive correlation among tropomodulins (TMODs) proteins expression and neuroblastoma progression. TMODs bind the pointed end of actin filaments, regulate polymerization and depolymerization processes modifying actin cytoskeletal dynamic and influencing neuronal development processes. Expression levels of TMODs genes were analyzed in 17 datasets comprising different types of tumors, including neuroblastoma, and it was demonstrated that high levels of tropomodulin1 (TMOD1) and tropomodulin 2 (TMOD2) correlate positively with high survival probability and with favorable clinical and molecular characteristics. Functional studies on neuroblastoma cell lines, showed that TMOD1 knockin induced cell cycle arrest, cell proliferation arrest and a mature functional differentiation. TMOD1 overexpression was responsible for particular cell morphology and biochemical changes which directed cells towards a neuronal favorable differentiation profile. TMOD1 downregulation also induced cell proliferation arrest but caused the loss of mature cell differentiation and promoted the development of neuroendocrine cellular characteristics, delineating an aggressive and unfavorable tumor behavior. Overall, these data indicated that TMODs are favorable prognostic biomarkers in neuroblastoma and we believe that they could contribute to unravel a new pathophysiological mechanism of neuroblastoma resistance contributing to the design of personalized therapeutics opportunities.

\section{INTRODUCTION}

Neuroblastoma is an embryonic tumor of the peripheral sympathetic nervous system, arising during fetal or early postnatal life from sympathetic cells derived from the neural crest. It is the most common solid extra cranial malignancy of childhood affecting 10.5 per million children between 0 and 14 years of age and it is responsible for $15 \%$ of all cancer-related mortalities in children $[1,2]$. Neuroblastoma is characterized by a broad range of clinical and biological heterogeneity, ranging from spontaneous regression or differentiation into benign tumors to rapid and aggressive phenotype that does not respond to current intensive multimodal therapy. In half of cases distant metastasis are detected at diagnosis and the tumor is classified as "high-risk" with a survival rate lower than $40 \%$ [3]. Several gene mutations, deletions or chromosomal rearrangements are involved in the onset of 
neuroblastoma and are associated with poor outcome, such as $A L K, P H O X 2 B$ and $M Y C N$ gene amplification [4, 5]. Our previous study identified Notch pathway as one of the molecular pathways involved in the onset and progression of neuroblastoma [6]. In particular, we recognized Notch ligand DLL1 as the specific molecular target in childhood neuroblastoma and we proposed miRNAs as novel therapeutic tool to attack "DLL1 positive" neuroblastoma [7]. The challenge is to identify innovative and selective biomarkers to better understand clinical and molecular mechanisms underlying neuroblastoma progression and to devise new personalized therapeutics opportunities. In this study we demonstrated a strong correlation between TMODs expression and neuroblastoma survival. TMODs are a conserved family of $40 \mathrm{kDa}$ proteins that cap actin filaments pointed end, stabilize filaments and inhibit their disassembly and turnover; they are defined pointed end capping protein for actin filaments [8, 9]. There are four TMOD isoforms (TMOD1, TMOD2, TMOD3, TMOD4) expressed in different tissues in vertebrates encoded by distinct genes. TMOD1 and TMOD2 isoforms are the only expressed in neurons, TMOD3 is ubiquitous and TMOD4 is mainly localized in skeletal muscle cells [10]. TMODs interact with a series of cytoskeletal proteins and this link allows to modulate the polymerization and depolymerization of actin monomers, modifying their dynamics and thus to act indirectly on the mechanical properties of the cytoskeletal and cellular physiology [11, 12]. Actin cytoskeletal dynamics play a crucial role in neuronal system development and drive important processes such as neurite extension, formation of axon, dendrites and growth cones migration [13, 14]. Fath and colleagues [15] demonstrated, for the first time, that TMOD1 and TMOD2 are negative regulators of neurite outgrowth and they have a key role in neurites formation and extension. TMODs can stabilize actin filaments that are not available for the formation of new neurites, or reduce the actin levels required for the polymerization. TMODs are also able to influence positively dendritic arbor, indeed overexpression of both TMOD1 and TMOD2 increased dendritic complexity and the branching [16]. Recently, TMODs are emerging as new protagonists in several diseases pathogenesis; TMOD2 has altered expression in fetal Down syndrome [17], mesial temporal lobe epilepsy [18], post-stroke [19] and post-methamphetamine exposure [20], while TMOD3 has been reported as a novel biomarker with high sensitivity and diagnostic accuracy in endometriosis [21]. Initial studies reported TMODs role in cancer; Kureha and colleagues [22] demonstrated that TMOD1 expression was directly regulated by NF- $\kappa \mathrm{B}$ and its overexpression was associated with enhanced breast tumor growth in a mouse xenograft model. TMOD1 is a powerful diagnostic marker for ALK-negative anaplastic large-cell lymphoma [23] and it was overexpressed frequently in oral squamous cell carcinoma [24]. TMOD2 high expression levels correlate with high survival probability and favorable disease outcome in neuroblastoma patients' [25], while TMOD3 up regulation is responsible for chemotherapeutic agents' resistance in non-small cell lung carcinoma [26]. Our data clearly demonstrated extensively a positive and specific correlation between TMOD1 and TMOD2 expression levels and neuroblastoma; high expression levels of these two genes were associated with high survival probability and good prognosis of neuroblastoma patients'. In vitro characterization and functional studies led us to better understand the role of TMOD1 in neuroblastoma cell lines. In particular, we identified that TMOD1 knockin caused cell cycle arrest, cell proliferation arrest in addition to a functional and mature cell differentiation. On the contrary, TMOD1 knockdown induced loss of expression of mature neuronal markers and the production of an unfavorable cell differentiation profile.

\section{RESULTS}

\section{TMOD1 and TMOD2 high expression levels are associated with high survival probability neuroblastoma patients'}

We assessed the expression of TMOD1 and TMOD2 genes in 17 different datasets comprising different types of tumors and we added an additional neuroblastoma dataset as control (Supplementary Table 1). We found that $T M O D s$ expression varied among tumor types but the highest expression of TMODI (Figure 1A) and TMOD2 (Figure 1B) was observed in neuroblastoma tumors (Bonf $\mathrm{p}<0.01$ ). We have not found a significant difference of expression for either genes between the two neuroblastoma datasets supporting the conclusion that either genes are highly expressed in neuroblastoma $(\mathrm{p}>0.05$, Figure 1A and 1B). These findings raised the question of the prognostic significance of these genes. We studied the survival curves of 498 neuroblastoma patients split in high or low TMODs expression. We found that 440 patients with high TMOD1 expression had a good overall survival (5y-OS \pm SE: $0.82 \pm 0.02 \%$ ) as opposed to 58 patients with low TMOD1 expression levels who had a poor overall survival $(5 \mathrm{y}-\mathrm{OS} \pm \mathrm{SE}$ : $0.44 \pm 0.07$ ) (Figure 1C). Likewise, 397 patients with high expression levels of TMOD2 had a good overall survival $(5 \mathrm{y}-\mathrm{OS} \pm \mathrm{SE}: 0.86 \pm 0.01)$ as opposed to 101 patients with low TMOD2 expression levels who had a poor overall survival (5y-OS \pm SE: $0.45 \pm 0.05 \%$ ) (Figure 1D). Overall survival between low and high expression of TMODs was significantly different (TMOD1 HR 0.27 95\%CI 0.06 0.23 and TMOD2 HR $0.1995 \%$ CI 0.04-0.13 both Bonf $\mathrm{p}<0.0001$ ). These results demonstrated that TMODs were prognostic and that good prognosis was associated with high expression levels. We confirmed the observation that TMOD1 and TMOD2 stratified patients into groups with different overall survival (TMOD1 HR $0.1595 \% \mathrm{CI}$ 0.02-0.14, and TMOD2 HR 0.06 95\% CI 0.02-0.1, both 
Bonf $\mathrm{p}<0.0001$ and TMOD1 HR 0.2 95\% CI 0.01-0.2, and TMOD2 HR 0.2 95\% CI 0.06-0.3, both Bonf $\mathrm{p}<0.0001$ ), and that high expression levels of TMOD1 and TMOD2 were associated with good prognosis on two independent datasets of 88 neuroblastoma primary tumors profiled by Affymetrix platform and 251 neuroblastoma primary tumors profiled by customized $11 \mathrm{~K}$ oligonucleotidemicroarrays (Supplementary Figure 1). Analysis of the relationship between TMODs expression and known neuroblastoma risk factors indicated that TMODs maintained a significant prognostic value when the model was adjusted for the clinical and molecular covariates (TMOD1 OS HR 0.4 95\% CI 0.2-0.7 and TMOD2 HR 0.5 ; 95\% CI 0.2-0.8, both $\mathrm{p} \leq 0.01$ ) (Supplementary Table 2) demonstrating that TMODs expression is an independent risk factors for neuroblastoma patients. Accordingly, TMOD1 and TMOD2 expression was significantly higher in low risk than in high risk patients. Higher TMODs expression was observed in patients with less than 18 months of age at diagnosis $(\mathrm{p}<0.01$; Figure $2 \mathrm{~A})$, in stage I, II, III, IVs tumors ( $<<0.01$; Figure $2 \mathrm{~B}$ ) and in $M Y C N$ single copy tumors $(\mathrm{p}<0.01$; Figure $2 \mathrm{C}$ ). These findings were confirmed in an independent dataset (Supplementary Figure 2) supporting the conclusion that TMODs expression is elevated in patients with favorable clinical and molecular characteristics.

\section{TMODs have different expression levels and cellular localization in differentiated neuroblastoma cells}

Neuroblastoma patients' data identified a positive correlation among TMODs high expression levels, good survival probability and favorable prognosis. On this basis, we investigated the role of TMODs in vitro on the biology of neuroblastoma cell lines. First, we evaluated the presence and the expression levels of TMOD1 and TMOD2 in SH-SY5Y and SK-N-SH neuroblastoma cells. Proteins and mRNA expression levels were studied in untreated and 13-cis retinoic acid treated cells, currently used in neuroblastoma therapy as cell-differentiating agent in association with standard therapeutic protocols [27]. Neuroblastoma cells were cultured for 72 hours after 13-cis retinoic acid treatment and then analyzed by Western Blotting and qRT-PCR. We observed, in both cell lines, that TMOD1 expression was barely detectable in the control group and significantly increased after 13-cis retinoic acid treatment; instead, TMOD2 protein expression level was present in untreated cells and did not increase after 13-cis retinoic acid treatment (Figure $3 \mathrm{~A}, 3 \mathrm{~B})$. These results are in agreement with the study of Fath and colleagues [15] in N2a neuroblastoma cells. To better-characterize TMODs behaviour, we studied the localization of these two proteins in neuroblastoma cell lines by immunocytochemistry. Staining TMODs in both cell lines, it emerged that TMOD1 has a cytoplasmic localization and it is expressed all along the cell neurites, while TMOD2 has a mainly perinuclear localization (Figure 3C).

\section{TMOD1 knockin induces cell cycle arrest in G0/ G1 phase and cell proliferation arrest}

Since both TMODs correlate positively with neuroblastoma and only TMOD1 expression levels increased significantly after 13 -cis retinoic acid treatment, we decided to investigate the effects of TMOD1 overexpression in neuroblastoma cell lines measured by cell cycle, proliferation and cell differentiation parameters. For cell cycle analysis, neuroblastoma cell lines were transfected with GFP-TMOD1 plasmid for 72 hours at three different concentrations $(1 \mu \mathrm{g} / \mu \mathrm{l}, 5 \mu \mathrm{g} / \mu \mathrm{l}, 10 \mu \mathrm{g} / \mu \mathrm{l})$. Flow cytometry analysis showed that TMOD1 knockin induced cell cycle arrest in G0/G1 phase (Figure 4A). In particular, there was a significant increase in the cells number in G0/G1 phase and a decrease of cells number in $\mathrm{S}$ phase cell cycle, compared to control. The data indicated that, in both cell lines, TMOD1 knockin blocked cells in $\mathrm{G} 0 / \mathrm{G} 1$ phase decreasing the tumor cells number in proliferation. The cell proliferation arrest was evaluated also by immunocytochemistry using cell proliferation marker Ki-67; TMOD1 knockin induced a significant cell proliferation arrest, in a dose dependent manner, with a decrease in the number of Ki-67 nuclei positive cells compared to the control (Figure 4B). A quantitative analysis of cell proliferation arrest was obtained by counting the number of $\mathrm{Ki}-67$ nuclei positive cells in a minimum of 10 fields for each treatment (Figure 4C). A direct nuclei cell count was also performed confirming a significant reduction of cells number induced by TMOD1 knockin compared to the control (mean cell number/field \pm S.E.M.: $70.0 \pm 8.375$ for control, $48.38 \pm 3.343$ for TMOD1 $1 \mu \mathrm{g} / \mu \mathrm{l}, 45.80 \pm 6.996$ for TMOD1 $5 \mu \mathrm{g} / \mu \mathrm{l}, 29.80$ \pm 6.689 for TMOD1 $10 \mu \mathrm{g} / \mu \mathrm{l})$.

\section{TMOD1 knockin induces in neuroblastoma cells a neuron-like differentiated profile}

Cells differentiation was analyzed in both cell lines by immunocytochemistry and representative images by confocal microscopy showed that TMOD1 knockin induced neuroblastoma cells differentiation characterized by long neurites with sprouting, growth cones and varicosities (Figure 5A). Neurites arborization and the formation of varicosities are the main features of functional and mature cell differentiation [28-30]. It was investigated in detail the biochemical implications of cell differentiation induced by TMOD1 knockin by evaluating the expression levels of a set of differentiation markers. qRT-PCR analysis showed a significant increase of neuronal markers such as Microtubule-associated protein 2 (MAP2) and Growth Associated protein 43 
(GAP43) (Figure 5B), while Tyrosine hydroxylase (TH), Dopamine beta-hydroxylase (DBH) and Chromogranin A ( $\mathrm{CgA})$ showed a decrease in mRNA expression levels (Figure 5C). MAP2 and GAP43 proteins are involved in neurogenesis and their expression is indicative of neuronal growth, development and stabilization. In conclusion, the morphological and biochemical data collected suggested that TMOD1 overexpression induced the development of a neuronal cell differentiation profile.

\section{TMOD1 knockdown induces in neuroblastoma cells a neuroendocrine differentiation profile}

To further examine the TMOD1 functional role in neuroblastoma cells, we studied the effects of TMOD1 downregulation utilizing RNA interference technique. TMOD1 downregulation, as occur with TMOD1 overexpression, induced both cell cycle and proliferation arrest (data not shown), but surprisingly it induced a new differentiation profile characterized by long and thin neurites, without sprouting, growth cones and varicosities (Figure 6A). The cell differentiation observed was very different compared to that obtained after TMOD1 overexpression. To better understand the nature of this type of differentiation, we evaluated the expression of a set of differentiation markers by qRT-PCR. After TMOD1 knockdown, Microtubule-associated protein 2 (MAP2) mRNA expression levels remained unchanged while there was a decrease of Growth Associated protein 43 (GAP43) neuronal marker (Figure 6B). Conversely, a significant
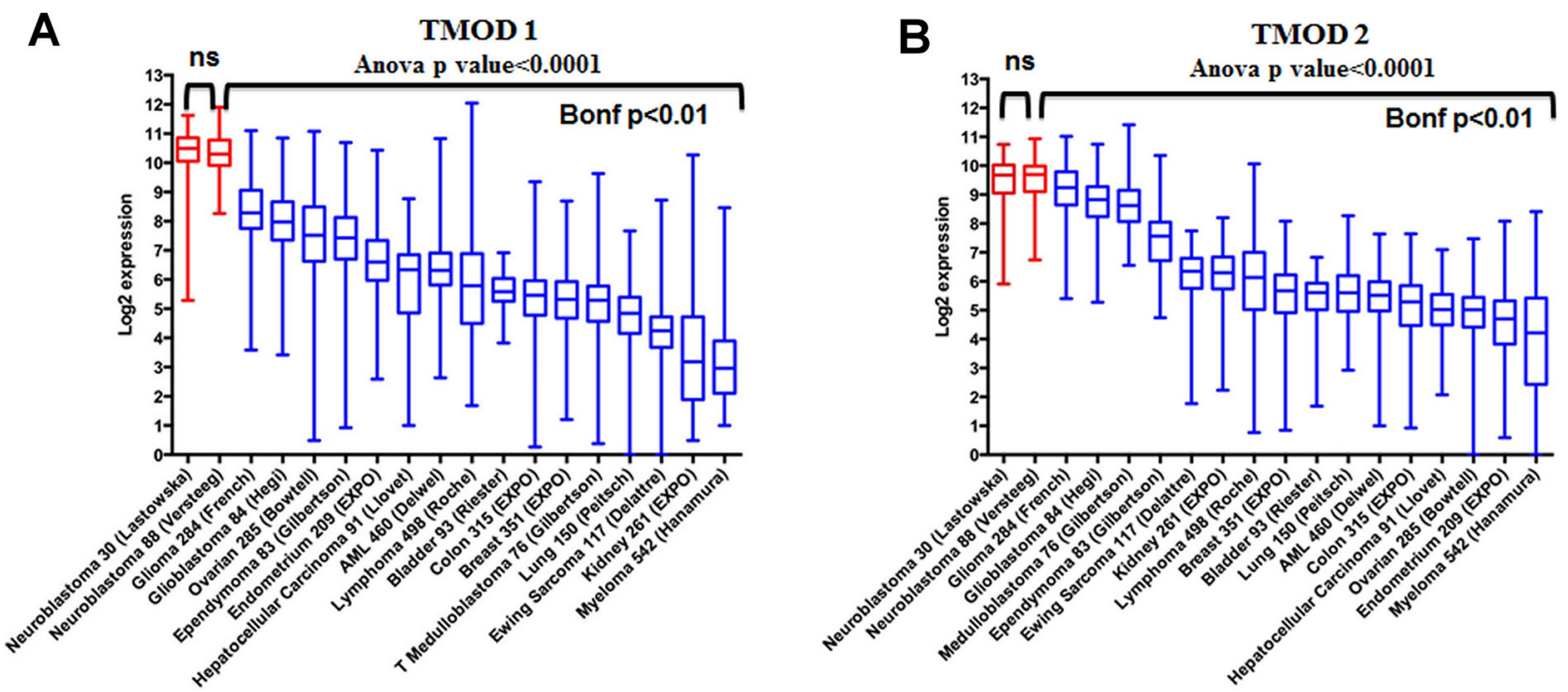

C

TMOD 1

D

TMOD 2
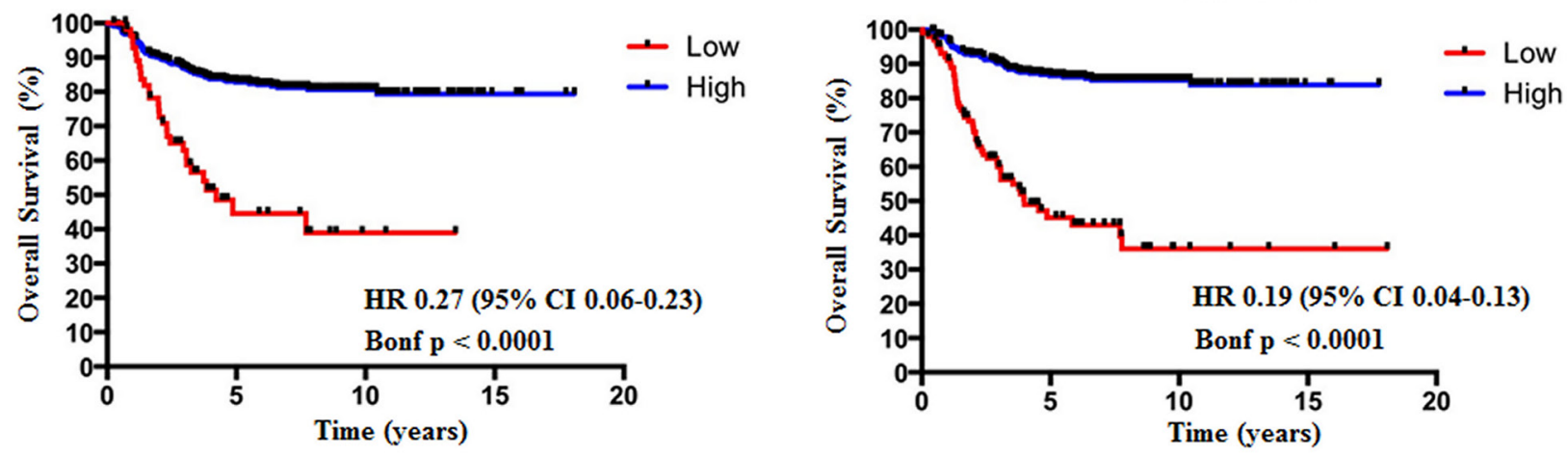

Figure 1: TMOD1 and TMOD2 genes are more expressed in neuroblastoma than in other tumors types and their high expression levels correlate with a good overall survival. The box and whisker plots show the expression of TMOD1 (A) and TMOD2 (B) in 18 different data sets. Red and blue boxes and whiskers refer to the neuroblastoma and other tumor types datasets, respectively. The box and whisker are sorted by decreasing order of median expression value. Plots are entitled with ANOVA p value and the corresponding gene symbol. The Bonferroni (Bonf) adjusted p value obtained by post-hoc analysis is reported below the black bar. We considered significant a $\mathrm{p}$ value $<0.05$. Kaplan-Meier curves show overall survival in the 498 patients' cohort stratified by $T M O D 1$ (C) and TMOD2 (D). Plots are entitled with the corresponding gene symbol. High expression (blue) and low expression (red) curves were compared by log-rank test and corrected for multiple hypotheses testing by Bonferroni method. Each plot reports the Bonferroni corrected $\mathrm{p}$ value (Bonf), the hazard ratio and confidence interval of the stratification. HR: hazard ratio. CI: confidence interval. ns: not significant. 

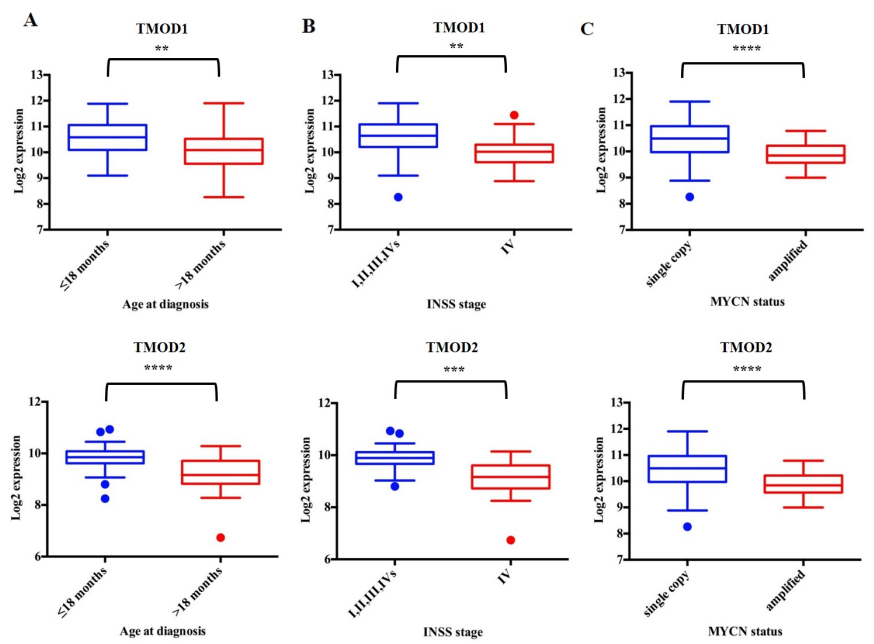

Figure 2: TMOD1 and TMOD2 expression levels correlate with favorable clinical and molecular characteristics. Box and whisker plots of TMOD1 and TMOD2 in the populations defined by Age at diagnosis (A), INSS stage (B) and MYCN status (C) risk factors. Data are relative to the patients in the 88 patients' cohort. Plots are entitled with the corresponding gene symbol. Significance of the difference between the means has been assessed by unpaired $t$ test. A probability smaller than 0.05 was considered a significant difference. ${ }^{* *}$ means $\mathrm{p}<0.001,{ }^{* * *}$ means $\mathrm{p}<0.0001$ and ${ }^{* * * *}$ means $\mathrm{p}<0.00001$. Age at diagnosis $(\leq 18$ months (blue) vs. $>18$ months (red)), INSS stage (I, II, III, IVs (blue) vs. IV(red)) and MYCN status (single copy (blue) vs. amplified (red)) groups define the clinical and molecular subpopulations.

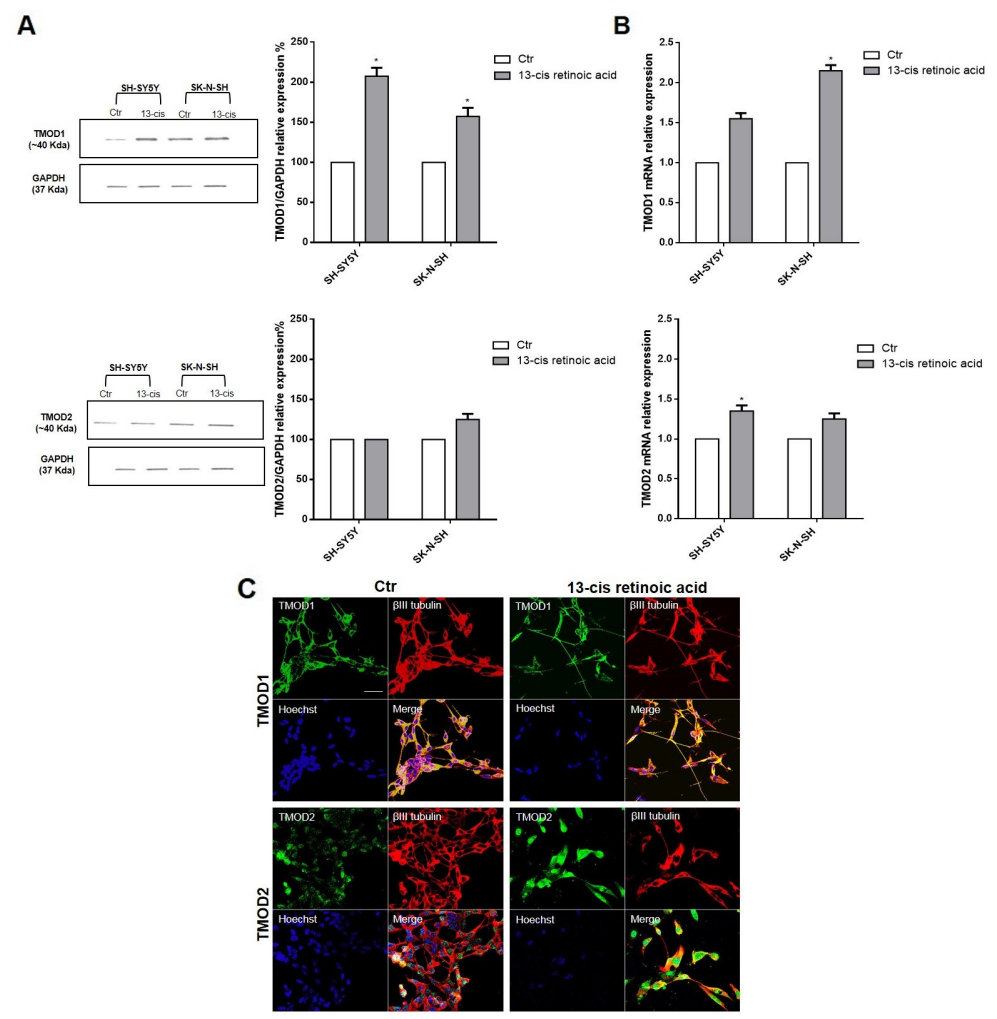

Figure 3: TMOD1 and TMOD2 have different expression levels and cellular localization in neuroblastoma cell lines. (A) Western Blotting analysis of TMOD1 and TMOD2 protein expression levels in SH-SY5Y and SK-N-SH untreated and 13-cis retinoic acid treated cells. GAPDH was used as a loading control. Densitometric analysis of TMOD1 and TMOD2 protein expression levels was obtained using Image Studio ${ }^{\text {TM }}$ Software- LI-COR System. (B) qRT-CR analysis of TMOD1 and TMOD2 mRNA expression levels in SHSY5Y and SK-N-SH untreated and 13-cis retinoic acid treated cells. Data are normalized to the GAPDH signal and are obtained from three experiments. * means $\mathrm{p}<0.05$ vs. control. (C) Representative confocal images of SH-SY5Y neuroblastoma cells after 72 hours in culture medium alone $(\mathrm{Ctr})$ or in medium added $40 \mu \mathrm{M}$ 13-cis retinoic acid. TMOD1 and TMOD2 antibodies were in green, $\beta$ III tubulin antibody was in red and Hoechst, used as nuclei marker, was in blue. Merge represents the superposition of the three signals. Scale bar, $40 \mu \mathrm{m}$. 

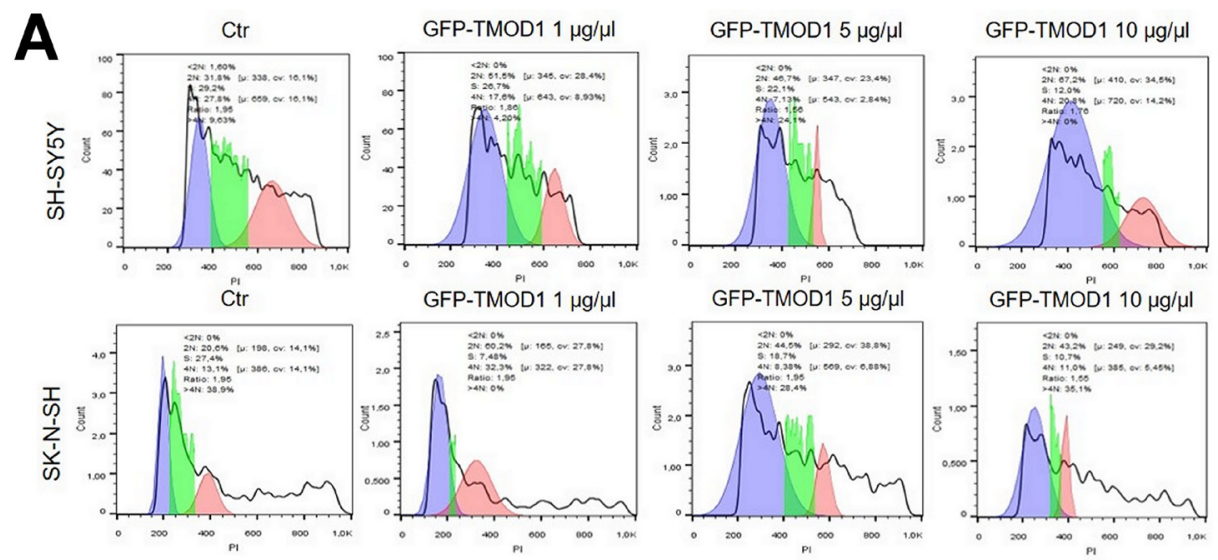

SH-SY5Y

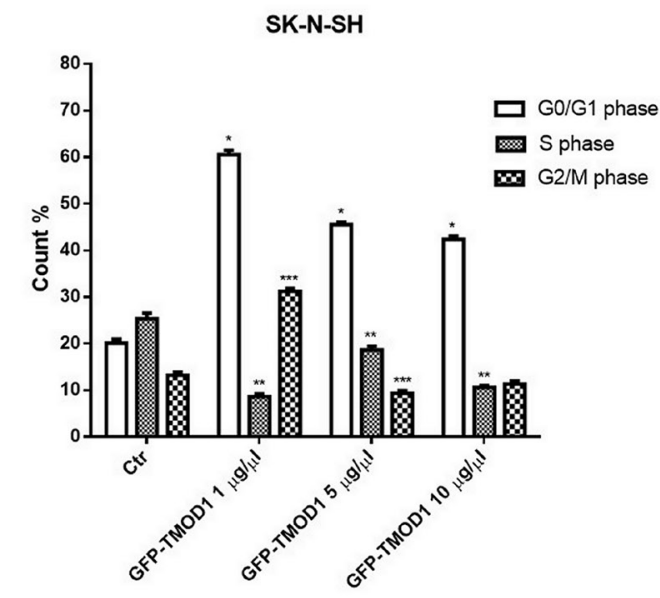

B
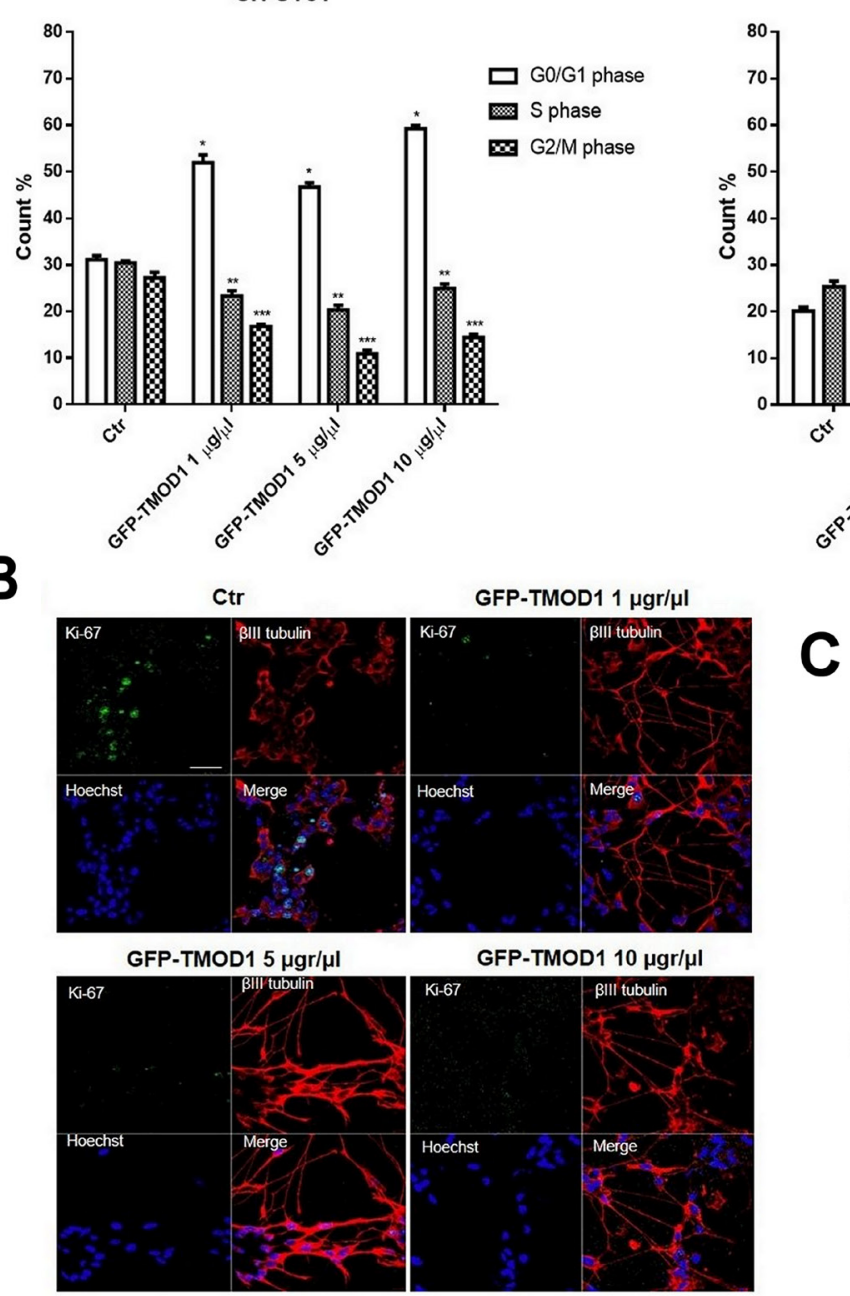

GFP-TMOD1 $10 \mu \mathrm{gr} / \mathrm{\mu l}$
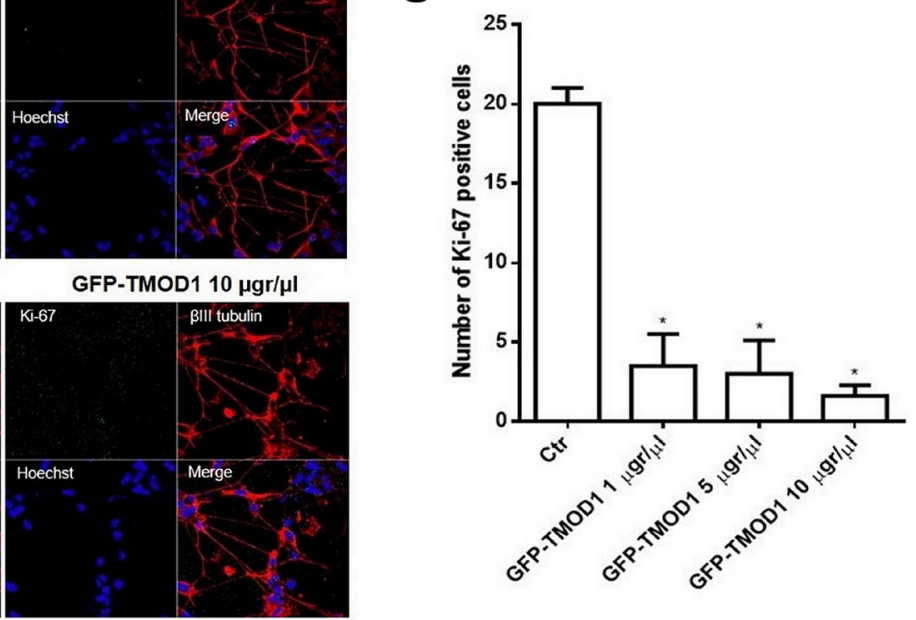

Figure 4: TMOD1 knockin induces cell cycle and cell proliferation arrest. (A) Cell cycle analysis of SH-SY5Y and SK-N-SH neuroblastoma cells transfected with GFP-TMOD1 plasmid for 72 hours with three different concentrations $(1 \mu \mathrm{g} / \mu \mathrm{l}, 5 \mu \mathrm{g} / \mu \mathrm{l}, 10 \mu \mathrm{g} / \mu \mathrm{l})$. "means $\mathrm{p}<0.0001$ vs. G0/G1 phase control; ${ }^{* *}$ means $\mathrm{p}<0.0001$ vs. S phase control; ${ }^{* * *}$ means $\mathrm{p}<0.0001$ vs. G2/M phase control. Data are obtained from three experiments. (B) Representative confocal images of SH-SY5Y neuroblastoma cells transfected with GFP-TMOD1 plasmid for 72 hours. Ki-67, proliferation marker, was in green, $\beta$ III tubulin antibody was in red and Hoechst, used as nuclei marker, was in blue. Merge represents the superposition of the three signals. Scale bar, $40 \mu \mathrm{m}$. (C) Quantitative analysis of proliferation cells was determined by counted the number of Ki- 67 nuclei positive cells at least 10 fields for each treatment. ${ }^{*}$ means $p<0.001$ vs. control. 
increase of Tyrosine hydroxylase (TH), Dopamine betahydroxylase (DBH), and Neuron-specific enolase (NSE) was observed (Figure 6C). In particular, we observed a significant increase of Chromogranin A (CgA) mRNA expression levels (Figure 6D), a neuroendocrine secretory protein, which is a useful prognostic and diagnostic tool for neuroblastoma and correlates with tumor burden and survival patients' [31]. These data suggest that TMOD1 downregulation induced the loss of neuron-like differentiated profile towards neuroendocrine profile.

\section{DISCUSSION}

The understanding of biological mechanisms and biomarkers involved in the onset and progression of neuroblastoma is needed to reach better prognostic stratification and more efficacious therapeutic strategies. In the present study, we demonstrated that TMODI and TMOD2 expression are independent prognostic markers and that high expression levels of these genes are associated with favorable outcome. Little is known about the relationship between neuroblastoma and TMOD genes. TMOD2 is part of a 160 gene signatures predicting neuroblastoma outcome [32] and of a 55 gene signature predicting neuroblastoma outcome in metastatic neuroblastoma lacking $M Y C N$ amplification [33]. There is no evidence of studies on TMOD1 and neuroblastoma. TMOD1 and TMOD2 were highly expressed in neuroblastoma, relative to other tumor types, suggesting an important role of these genes in the tumor homeostasis. Moreover, both genes showed a strong association between high expression and favorable neuroblastoma prognosis. The strength of this result relies on the large number of samples in the three neuroblastoma patients' datasets (837 tumor samples) that have been used to reach this conclusion;
A

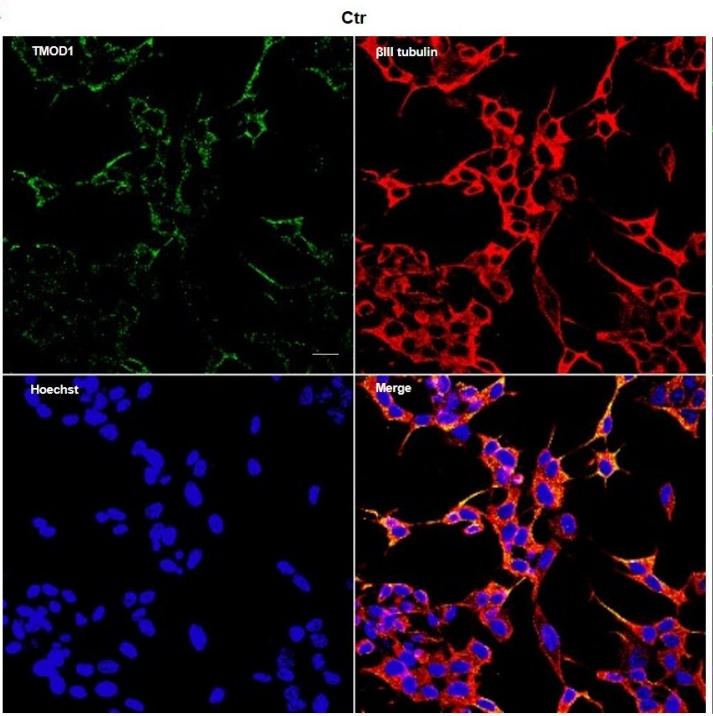

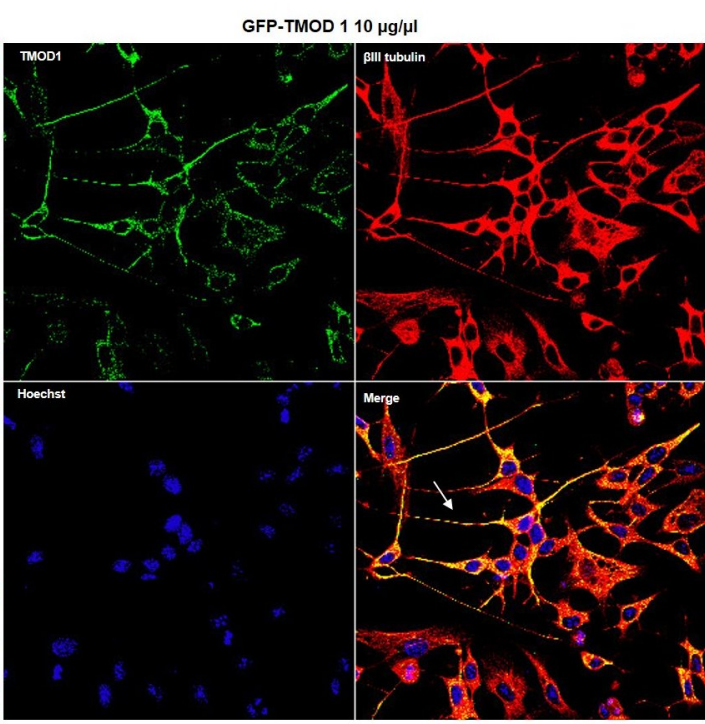

C

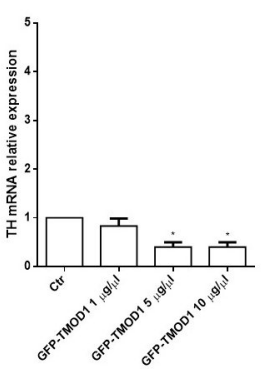

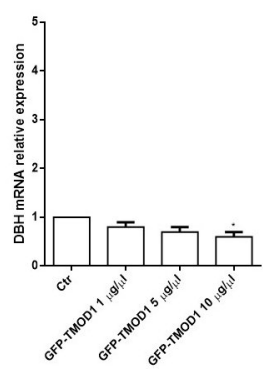

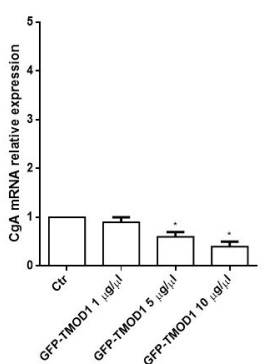

Figure 5: TMOD1 knockin induces in neuroblastoma cells a neuron-like differentiated profile. (A) Representative confocal images of SH-SY5Y neuroblastoma cells transfected with GFP-TMOD1 plasmid at the concentration of $10 \mu \mathrm{g} / \mu \mathrm{l}$ for $72 \mathrm{hours}$. TMOD1 was in green, $\beta$ III tubulin antibody was in red and Hoechst, used as nuclei marker, was in blue. Merge represents the superposition of the three signals. Arrow indicates neurite branching and varicosities. Scale bar, $40 \mu \mathrm{m}$. (B) qRT-PCR analysis of MAP2 and GAP43 mRNA expression levels in SH-SY5Y untreated and GFP-TMOD1 plasmid transfected cells. (C) qRT-PCR analysis of TH, DBH and CgA mRNA expression levels in SH-SY5Y untreated and GFP-TMOD1 plasmid transfected cells. Data are normalized to the GAPDH signal and are obtained from three experiments. " means $\mathrm{p}<0.05$ vs. control. 
this conclusion was further supported by the observation that newborn patients, no $M Y C N$ amplification, or low grade stage had a higher expression of TMOD1 and TMOD2 in two independent datasets. In vitro functional studies demonstrated that TMOD1 knockin induced cell cycle arrest in G0/G1 phase, cell proliferation arrest, a functional and mature neuron-like differentiation. The biochemical differentiation profile of neuroblastoma cells overexpressing TMODs showed a significant increase of MAP2 and GAP43, mimicking then the "neuronal differentiation" obtained by retinoic acid, and an overall decrease of TH, DBH and CgA mRNA expression levels. On the contrary, TMOD1 downregulation caused the loss of mature and functional differentiation cell profile, with the appearance of long and thin neurites without sprouting and varicosities, the accumulation sites of the secretory vesicles of neurotransmitters that are released in response to neurosecretory stimuli $[29,30]$. Biochemical analysis evidenced a decrease of neuronal biomarkers expression and a significant increase of $\mathrm{TH}, \mathrm{DBH}, \mathrm{NSE}$ and $\mathrm{CgA}$, indexes of neuroendocrine differentiation and considered unfavorable prognostic neuroblastoma biomarkers [34]. In a recent study, Lee and colleagues [35] evaluated the clinical significant of TH mRNA transcripts levels in the peripheral blood of patients with neuroblastoma demonstrating that $\mathrm{TH}$ expression was associated with high-risk features, advanced stage and worse outcome. Previous studies also reported that positive $\mathrm{TH}$ expression was a poor prognostic indicator in metastatic neuroblastoma [36, 37], suggesting a role of this marker in stratification. In particular we observed an increase of NSE by tenfold and $\mathrm{CgA}$ mRNA expression
A

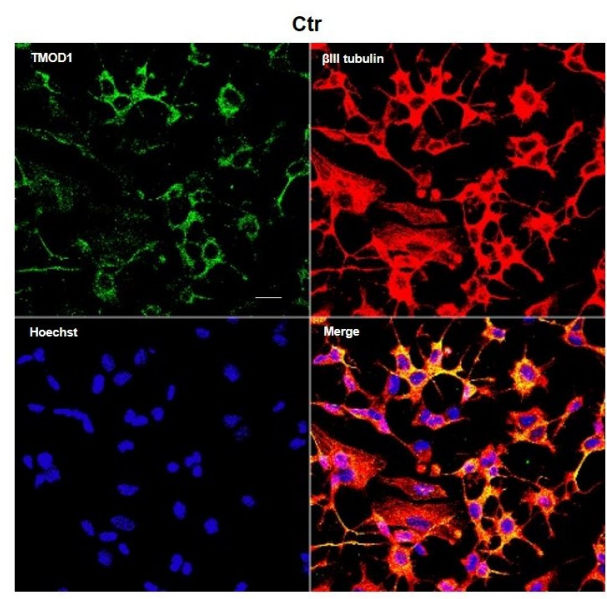

SIRNA 5 TMOD1

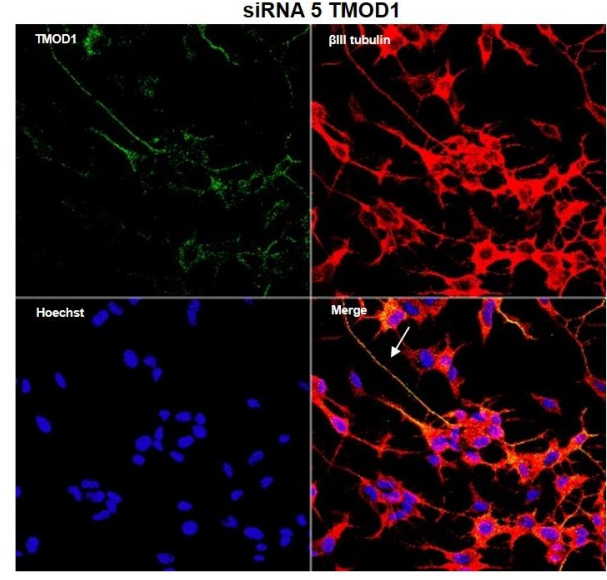

B
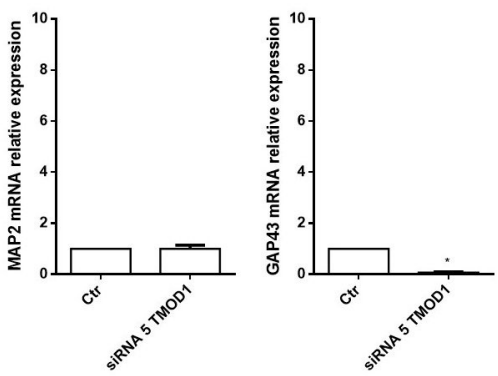

C
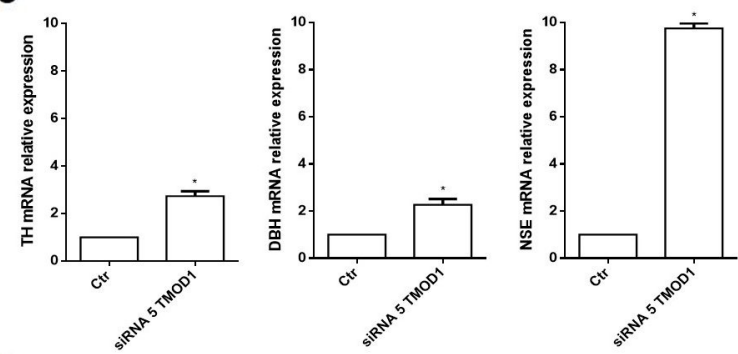

D

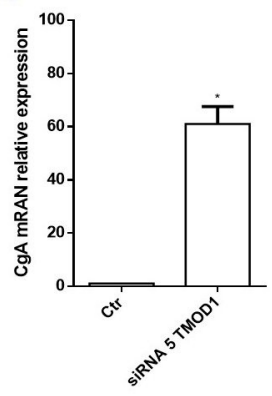

Figure 6: TMOD1 knockdown induces in neuroblastoma cells a neuroendocrine differentiation profile. (A) Representative confocal images of siRNA 5 TMOD1 downregulation effects in SH-SY5Y neuroblastoma cells after 72 hours of transfection. TMOD1 was in green, $\beta$ III tubulin antibody was in red and Hoechst, used as nuclei marker, was in blue. Merge represents the superposition of the three signals. Arrow indicates thin neurite without varicosities. Scale bar, $40 \mu \mathrm{m}$. (B) qRT-PCR analysis of MAP2 and GAP43 mRNA expression levels in SH-SY5Y untreated and TMOD1 knockdown treated cells. (C) qRT-PCR analysis of TH, DBH and NSE mRNA expression levels in SH-SY5Y untreated and TMOD1 knockdown treated cells. (D) qRT-PCR analysis of CgA mRNA expression levels in SH-SY5Y untreated and TMOD1 knockdown treated cells. Data are normalized to the GAPDH signal and are obtained from three experiments. * means $\mathrm{p}<0.05$ vs. control. 
sixty-fold higher than control. $\mathrm{CgA}$ is a pro-hormone that is stored in the same vesicles and co-released with catecholamines in adrenal medulla and postganglionic sympathetic axons and regulates in turn chatecolamines secretion with a negative feedback mechanism [38]. NSE and $\mathrm{CgA}$ expression correlate with the differentiation level of the tumor, metastatic sites and response to treatment; patients in stage I and stage II have low levels of $\mathrm{CgA}$ and favorable outcome, while patients with advanced disease stages (III and IV) have high levels of NSE and CgA and bad prognosis $[39,40]$. Furthermore hypoxia, validated as predictor of poor outcome in neuroblastoma, was found to induce a shift from neuronal to chromaffin cell differentiation, with high expression of neuroendocrine markers and decrease in neuronal markers as GAP43 [41]. Finally, GSK inhibitors were found to arrest neuroblastoma cells proliferation coupled with a decrease in neuroendocrine markers [42]. Thus, in our experimental data, TMOD1 knockdown induced high expression levels of all unfavorable neuroendocrine biomarkers, delineating an aggressive tumor profile. In conclusion, TMODs are able to influence positively survival probability and prognosis of neuroblastoma patients mainly acting on cell differentiation. Sympathetic neurons and chromaffin cells derive from a common fate-restricted sympathoadrenal progenitor of the neural crest, which, in response to the local environment and a network of transcription factors, can choose one or the other differentiation path [43]. Thus, TMODs could represents, in prenatal and early postnatal development, a signal for the correct differentiation of sympathetic cells. Although further investigation is required, these data identify a new pathway involved in neuroblastoma and therefore new strategies will develop to tackle the tumor.

\section{MATERIALS AND METHODS}

\section{Gene expression datasets}

Three publically available datasets were used for gene expression analysis. The first dataset [44] contains the gene expression profile of 498 neuroblastomas measured by the Illumina HiSeq 2000 RNAseq platform (GSE62564). The second one [45] contains the gene expression profile of 88 tumors measured by the Affymetrix Human Genome U133 Plus 2.0 platform (GSE16476). The third one [Customized oligonucleotide microarray gene expression-based classification of neuroblastoma patients outperforms current clinical risk stratification. Journal of Clinical Oncology, $24,5070-5078$ ] contains the gene expression profile of 251 tumors generated as dye-flipped dual-color replicates using customized $11 \mathrm{~K}$ oligonucleotide-microarrays (E-TABM-38). In addition, we utilized 3929 publically available expression profiles from 17 different tumor types by the Affymetrix Human Genome U133 Plus 2.0 platform and stored in the R2: Genomic Analysis and Visualization
Platform (http://r2.amc.nl). Good and poor outcome were defined as the patient's alive or dead status 5 years after diagnosis.

\section{Cell culture}

The human SH-SY5Y neuroblastoma cell line was cultured in a 1:1 mixture of Ham's F12 nutrient and Dulbecco's modified Eagle's medium (Sigma-Aldrich) supplemented with $10 \%$ fetal bovine serum (FBS, SigmaAldrich), $2 \mathrm{mM} \mathrm{L}$-glutamine, $50 \mathrm{mg} / \mathrm{mL}$ penicillin, and $100 \mathrm{mg} / \mathrm{mL}$ streptomycin (Sigma-Aldrich). The SK-NSH neuroblastoma cell line was cultured in Dulbecco's modified Eagle's medium (Sigma-Aldrich) supplemented with $10 \%$ fetal bovine serum (FBS, Sigma-Aldrich), 2 $\mathrm{mM} \mathrm{L}$-glutamine, $50 \mathrm{mg} / \mathrm{mL}$ penicillin, and $100 \mathrm{mg} /$ $\mathrm{mL}$ streptomycin (Sigma-Aldrich). All the cell lines were grown at $37^{\circ} \mathrm{C}$ in a $95 \%$ air- $5 \% \mathrm{CO}_{2}$ humidified incubator. Cell lines, at passage lower than 10 , were used within 3 months after receipt. Cells supplied by ECACC, the European Collection of Authenticated Cell Cultures, undergo quality control and authentication procedures. These include testing for mycoplasma by culture isolation, Hoechst DNA staining and PCR, together with culture testing for contaminant bacteria, yeast and fungi. Authentication procedures used include species verification by DNA barcoding and identity verification by DNA profiling. Human cell lines are analyzed by PCR of short tandem repeat sequences within chromosomal microsatellite DNA (STR-PCR).

\section{3-cis Retinoic acid treatment}

Twenty-four hours after seeding in complete media, neuroblastoma cell lines were cultured with $40 \mu \mathrm{M} 13-$ cis retinoic acid (Sigma-Aldrich). At $80 \%$ confluence, after 72 hours, cells were collected and proteins were extracted for Western Blotting analysis or fixed for immunofluorescence and morphologic analysis.

\section{DNA plasmid amplification and transient transfection}

GFP-TMOD1 plasmid was processed with bacterial transformation and amplification. The GFPTMOD1 plasmid was purified by Plasmid Midiprep Kit (Sigma-Aldrich), according to the manufacturer's protocol. SH-SY5Y and SK-N-SH neuroblastoma cell lines were transfected with the plasmid, at three different concentrations, using Lipofectamine reagent 2000 (Invitrogen). The complete process is indicated in Supplementary Materials and Methods.

\section{siRNA transient transfection}

The human-specific TMOD1 interference was performed assessing the efficacy of four different siRNAs 
at different concentrations to evaluate which siRNA was able to induce a greater downregulation of TMOD1 mRNA expression levels (Supplementary Figure 3). SH-SY5Y and SK-N-SH neuroblastoma cell lines were transfected with siRNA 5 at the concentration of $10 \mathrm{nM}$ using Hi-perfect transfection reagent (Qiagen) in culture medium 1 day after seeding. Cells were cultured for 72 hours after transfection with siRNA 5 and then collected for the mRNA expression levels analysis.

\section{RNA extraction and quantitative RT- PCR}

The total RNA was isolated from SH-SY5Y and SK-N-SH neuroblastoma cells using the RNeasy kit (Qiagen) and digested with the RNase-Free DNase set (Qiagen), according to the manufacturer's protocol. RNA was retrotranscribed using murine leukemia virus reverse transcriptase (Promega Italia) and oligo (dT) 15-18 as a primer. Quantitative RT-PCR (qRT-PCR) was performed by the SYBR Green I system (BioRad Italy) and detection were performed with the ViiA $7^{\mathrm{TM}}$ RT-PCR System (Applied Biosystems). Primers and complete protocol are indicated in Supplementary Materials and Methods.

\section{Western blotting}

Total cell lysates were prepared by scraping the cells in lysis buffer. $15 \mu \mathrm{g}$ of total proteins were electrophoresed into $10 \%$ SDS-PAGE precast gel, transferred to nitrocellulose paper, and stained with the antibodies of interest, followed by the appropriate secondary antibodies. Antibodies and full protocols are listed in Supplementary Materials and Methods.

\section{Immunofluorescence analysis}

Neuroblastoma cells were plated with a density of $75 \times 10^{3} /$ well in a 24 wells plate, fixed in ice-cold methanol, washed and incubated in Phosphate Buffered Saline and Bovine Serum Albumin. Cells were stained with the primary antibodies of interest, overnight at $4^{\circ} \mathrm{C}$, followed by the secondary antibodies conjugated with Alexa Fluor ${ }^{\circledR} 488$ or CYTM3. Antibodies and full protocols are listed in Supplementary Materials and Methods.

\section{Flow cytometry analysis}

For cell cycle analysis, cells were harvested at the completion of the siRNA 5 TMOD1 and GFP-TMOD1 transfections and washed with phosphate-buffered saline (PBS; pH 7.4) before being fixed with $70 \%$ ethanol on the wheel for 15 minutes at $4^{\circ} \mathrm{C}$. Subsequently, the cells were centrifuged at $4500 \mathrm{rpm}$ for 5 minutes at $4^{\circ} \mathrm{C}$, washed with phosphate-buffered saline and cells were resuspended in $600 \mu \mathrm{l}$ of $0,1 \%$ sodium citrate (Sigma-Aldrich), $50 \mu \mathrm{g} /$ $\mathrm{ml}$ of propidium iodide (PI, Sigma-Aldrich) and $10 \mu \mathrm{g} / \mathrm{ml}$ of Ribonuclease A (Sigma-Aldrich) for staining cellular
DNA. The cellular DNA content was then analyzed using a MACS Quant Flow Cytometer (Miltenyi Biotech). Data analysis was carried out using FlowJo software V10.

\section{Bioinformatics and statistical analysis}

Log2-transformed gene expression values were utilized whenever possible. Gene symbol was selected as reference annotation. In the Affymetrix platform the highest probe set expression was associated to the gene symbol [45]. Analysis was carried out, in part by the R2: Genomic Analysis and Visualization Platform (http:// r2.amc.nl). Patients overall survival (OS) was assessed by Kaplan-Meier curves. Survival probability was reported as 5-years overall survival \pm standard error $(5 y-O S \pm S E)$. Log-rank test, corrected for multiple hypotheses testing by the Bonferroni method, assessed the significance of the separation between survival curves. Multivariate analysis with a Cox proportional regression model evaluated the relationship among prognostic factors. The validity of the proportional hazards assumption was tested via evaluation of scaled Schoenfeld residuals. The significance of the difference between mean values was determined by paired or unpaired $t$-test or by ANOVA Kruskal-Wallis test when the comparison involved more than two groups with significantly different standard deviation.

\section{Author contributions}

Paola Bettinsoli, Giulia Ferrari-Toninelli and Maurizio Memo designed the study; Paola Bettinsoli, Giulia Ferrari-Toninelli, Maurizio Memo, Davide Cangelosi and Luigi Varesio developed the methodology; Paola Bettinsoli, Sara Anna Bonini, Michela Guarienti and Davide Cangelosi conducted experiments and performed data analysis; Paola Bettinsoli and Giulia FerrariToninelli wrote the manuscript; Sara Anna Bonini, Davide Cangelosi, Luigi Varesio and Maurizio Memo revised the manuscript by providing comments and suggestions; Maurizio Memo supervised the manuscript.

\section{ACKNOWLEDGMENTS}

The authors thank Prof. Velia M. Fowler of the Scripps Research Institute of California for GFP-TMOD1 plasmid.

\section{CONFLICTS OF INTEREST}

The authors declare that they have no conflicts of interest.

\section{FUNDING}

Funding for this work was provided by Institutional Research Grants from the University of Brescia (2015 No. 
8857 and 2016 HW-REFRACT) and Grant from Italian Neuroblastoma Foundation (project title: Clinical activity in Neuroblastoma).

\section{REFERENCES}

1. Maris JM, Hogarty MD, Bagatell RL, Cohn S. Neuroblastoma. Lancet. 2007; 369:2106-20.

2. Davidoff AM. Neuroblastoma. Semin Pediatr Surg. 2012; 21:2-14.

3. Garaventa A, Perilongo G. High risk neuroblastoma: a persistent therapeutic challenge. Pediatr Blood Cancer. 2008; 51:722-3.

4. Irwin MS, Park JR. Neuroblastoma: paradigm for precision medicine. Pediatr Clin North Am. 2015; 62:225-56.

5. Tang XX, Zhao H, Kung B, Kim DY, Hicks SL, Cohn SL, Cheung NK, Seeger RC, Evans AE, Ikegaki N. The MYCN Enigma: significance of MYCN expression in neuroblastoma. Cancer Res. 2006; 66:2826-33.

6. Ferrari-Toninelli G, Bonini SA, Uberti D, Buizza L, Bettinsoli P, Poliani PL, Facchetti F, Memo M. Targeting Notch pathway induces growth inhibition and differentiation of Neuroblastoma. Neuro Oncol. 2010; 12:1231-43.

7. Bettinsoli P, Ferrari-Toninelli G, Bonini SA, Prandelli C, Memo M. Notch ligand Delta-like 1 as a novel molecular target in childhood Neuroblastoma. BMC Cancer. 2017; 17:352.

8. Yamaschiro S, Gokhin DS, Kimura S, Nowak RB, Fowler VM. Tropomodulins: pointed-end capping proteins that regulate actin filament architecture in diverse cell types. Cytoskeleton (Hoboken). 2012; 69:337-370.

9. Fischer RS, Fowler VM. Tropomodulins: life at the slow end. Trends Cell Biol. 2003; 13:593-601.

10. Cox PR, Zoghbi HY. Sequencing, expression analysis, and mapping of three unique human tropomodulin genes and their mouse orthologs. Genomics. 2000; 63:97-107.

11. Colpan M, Moroz NA, Kostyukova AS. Tropomodulins and tropomyosins: working as a team. J Muscle Res Cell Motil. 2013; 34:247-260.

12. Kostyukova AS. Tropomodulins and Tropomodulin/ tropomyosin interactions. Cell Mol Life Sci. 2008; 65:563-569.

13. Gray KT, Kostyukova AS, Fath T. Actin regulation by tropomodulin and tropomyosin in neuronal morphogenesis and function. Mol Cell Neurosci. 2017; 84:48-57.

14. Dent EW, Gupton SL, Gertler FB. The growth cone cytoskeleton in axon outgrowth and guidance. Cold Spring Harb Perspect Biol. 2011; 3:3.

15. Fath T, Fischer RS, Dehmelt L, Halpain S, Fowler VM. Tropomodulins are negative regulators of neurite outgrowth. Eur J Cell Biol. 2011;90:291-300.

16. Gray KT, Suchowerska AK, Bland T, Colpan M, Wayman G, Fath T, Kostyukova AS. Tropomodulin isoforms utilize specific binding functions to modulate dendrite development. Cytoskeleton (Hoboken). 2016; 73:316-28.

17. Sun Y, Dierssen M, Toran N, Pollak DD, Chen WQ, Lubec G. A gel-based proteomic method reveals several protein pathway abnormalities in fetal Down syndrome brain. $\mathrm{J}$ Proteomics. 2011; 74:547-557.

18. Yang JW, Czech T, Felizardo M, Baumgartner C, Lubec G. Aberrant expression of cytoskeleton proteins in hippocampus from patients with mesial temporal lobe epilepsy. Amino Acids. 2006; 30:477-493.

19. Chen A, Liao WP, Lu Q, Wong WS, Wong PT. Upregulation of dihydropyrimidinase- related protein 2, spectrin alpha II chain, heat shock cognate protein 70 pseudogene 1 and tropomodulin 2 after focal cerebral ischemia in rats -a proteomics approach. Neurochem Int. 2007; 50:1078-1086.

20. Iwazaki T, McGregor IS, Matsumoto I. Protein expression profile in the striatum of acute methamphetamine-treated rats. Brain Res. 2006; 1097:19-25.

21. Gajbhiye R, Bendigeri T, Ghuge A, Bhusane K, Begum S, Warty N, Sawant R, Padte K, Humane A, Dasmahapatra P, Chauhan A, Khan S. Panel of autoimmune markers for noninvasive diagnosis of minimal-mild endometriosis: a multicenter study. Reprod Sci. 2017; 24:413-420.

22. Ito-Kureha $\mathrm{T}$, Koshikawa N, Yamamoto $\mathrm{M}$, Semba $\mathrm{K}$, Yamaguchi $\mathrm{N}$, Yamamoto $\mathrm{T}$, Seiki $\mathrm{M}$, Inoue $\mathrm{J}$. Tropomodulin 1 expression driven by NF- $\mathrm{B}$ enhances breast cancer growth. Cancer Res. 2015; 75:62-72.

23. Agnelli L, Mereu E, Pellegrino E, Limongi T, Kwee I, Bergaggio E, Ponzoni M, Zamò A, Iqbal J, Piccaluga PP, Neri A, Chan WC, Pileri S, and European T-Cell Lymphoma Study Group. Identification of a 3-gene model as a powerful diagnostic tool for the recognition of ALK-negative anaplastic large-cell lymphoma. Blood. 2012; 120:1274-81.

24. Suzuki T, Kasamatsu A, Miyamoto I, Saito T, Higo M, Endo-Sakamoto Y, Shiiba M, Tanzawa H, Uzawa K. Overexpression of TMOD1 is associated with enhanced regional lymph node metastasis in human oral cancer. Int $\mathbf{J}$ Oncol. 2016; 48:607-12.

25. Middelbeek J, Vrenken K, Visser D, Lasonder E, Koster J, Jalink K, Clark K, van Leeuwen FN. The TRPM7 interactome defines a cytoskeletal complex linked to neuroblastoma progression. Eur J Cell Biol. 2016; 95:465-474.

26. Paul D, Chanukuppa V, Reddy PJ, Taunk K, Adhav R, Srivastava S, Santra MK, Rapole S. Global proteomic profiling identifies etoposide chemoresistance markers in non-small cell lung carcinoma. J Proteomics. 2016; 138:95-105.

27. Reynolds CP, Matthay KK, Villablanca JG, Maurer BJ. Retinoid therapy of high-risk neuroblastoma. Cancer Lett. 2003; 197:185-92.

28. De Paola V, Arber S, Caroni P. AMPA receptors regulate dynamic equilibrium of presynaptic terminals in mature hippocampal networks. Nat Neurosci. 2003; 6:491-500. 
29. De Paola V, Holtmaat A, Knott G, Song S, Wilbrecht L, Caroni P, Svoboda K. Cell type-specific structural plasticity of axonal branches and boutons in the adult neocortex. Neuron. 2006; 49:861-875.

30. Ferrari-Toninelli G, Bonini SA, Uberti D, Napolitano F, Stante M, Santoro F, Minopoli G, Zambrano N, Russo T, Memo M. Notch activation induces neurite remodeling and functional modifications in SH-SY5Y neuronal cells. Dev Neurobiol. 2009; 69:378-91.

31. Hsiao RJ, Seeger RC, Yu AL, O'Connor D. Chromogranin $\mathrm{a}$ in children with neuroblastoma. J Clin Invest. 1990; 85:1555-59.

32. Chen QR, Song YK, Wei JS, Bilke S, Asgharzadeh S, Seeger RC, Khan J. An integrated cross-platform prognosis study on neuroblastoma patients. Genomics. 2008; 92:195-203.

33. Asgharzadeh S, Pique-Regi R, Sposto R, Wang H, Yang Y, Shimada H, Matthay K, Buckley J, Ortega A, Seeger RC. Prognostic significance of gene expression profiles of metastatic neuroblastomas lacking MYCN gene amplification. J Natl Cancer Inst. 2006; 98:1193-203.

34. Molenaar WM, Baker DL, Pleasure D, Lee VM, Trojanowski JQ. The neuroendocrine and neural profiles of neuroblastomas, ganglioneuroblastomas, and ganglioneuromas. Am J Pathol. 1990; 136:375-82.

35. Lee NH, Son MH, Choi YB, Yi E, Lee JW, Yoo KH, Sung KW, Koo HH. Clinical Significance of Tyrosine Hydroxylase mRNA transcripts in peripheral blood at diagnosis in patients with neuroblastoma. Cancer Res Treat. 2016; 48:1399-407.

36. Burchill SA, Lewis IJ, Abrams KR, Riley R, Imeson J, Pearson AD, Pinkerton R, Selby P. Circulating neuroblastoma cells detected by reverse transcriptase polymerase chain reaction for tyrosine hydroxylase mRNA are an independent poor prognostic indicator in stage 4 neuroblastoma in children over 1 year. J Clin Oncol. 2001; 19:1795-801.

37. Viprey VF, Gregory WM, Corrias MV, Tchirkov A, Swerts K, Vicha A, Dallorso S, Brock P, Luksch R, ValteauCouanet D, Papadakis V, Laureys G, Pearson AD, et al. Neuroblastoma mRNAs predict outcome in children with stage 4 neuroblastoma: a European HR-NBL1/SIOPEN study. J Clin Oncol. 2014; 32:1074-83.

38. Pasqua T, Mahata S, Bandyopadhyay GK, Biswas A, Perkins GA, Sinha-Hikim AP, Goldstein DS, Eiden LE, Mahata SK. Impact of Chromogranin A deficiency on catecholamine storage, catecholamine granule morphology and chromaffin cell energy metabolism in vivo. Cell Tissue Res. 2016; 363:693-712.

39. Isgrò $\mathrm{M}$, Bottoni $\mathrm{P}$, Scatena R. Neuron-specific enolase as a biomarker: biochemical and clinical aspects. Adv Exp Med Biol. 2015; 867:125-43.

40. Nowicki M, Ostalska-Nowicka D, Miskowiak B. Prognostic value of satge IV neuroblastoma metastatic immunophenotype in the bone marrow: preliminary report. J Clin Pathol. 2006; 59:150-152.

41. Hedborg F, Fischer-Colbrie R, Ostlin N, Sandstedt B, Tran MG, Maxwell PH. Differentiation in neuroblastoma: diffusion-limited hypoxia induces neuro-endocrine secretory protein 55 and other markers of a chromaffin phenotype. PLoS One. 2010; 5:e12825.

42. Carter YM, Kunnimalaiyaan S, Chen H, Gamblin TC, Kunnimalaiyaan M. Specific glycogen synthase kinase-3 inhibition reduces neuroendocrine markers and suppresses neuroblastoma cell growth. Cancer Biol Ther. 2014; 15:510-515.

43. Huber K. The sympathoadrenal cell lineage: Specification, diversification, and new perspectives. Dev Biol. 2006; 298:335-43.

44. Zhang W, Yu Y, Hertwig F, Thierry-Mieg J, Zhang W, Thierry-Mieg D, Wang J, Furlanello C, Devanarayan V, Cheng J, Deng Y, Hero B, Hong H, et al. Comparison of RNA-seq and microarray-based models for clinical endpoint prediction. Genome Biol. 2015; 16:133.

45. Molenaar JJ, Koster J, Zwijnenburg DA, van Sluis P, Valentijn LJ, van der Ploeg I, Hamdi M, van Nes J, Westerman BA, van Arkel J, Ebus ME, Haneveld F, Lakeman A, et al. Sequencing of neuroblastoma identifies chromothripsis and defects in neuritogenesis genes. Nature. 2012; 483:589-93. 